\title{
Publisher's Note: Approaching ultrastrong coupling in transmon circuit QED using a high-impedance resonator [Phys. Rev. B 95, 224515 (2017)]
}

Sal J. Bosman, Mario F. Gely, Vibhor Singh, Daniel Bothner, Andres Castellanos-Gomez, and Gary A. Steele

(Received 19 December 2018; published 4 January 2019)

DOI: 10.1103/PhysRevB.99.019901

This paper was published online on 29 June 2017 with omissions in the Acknowledgments on page 4. On page 4, the Acknowledgments should read as "The authors thank Alessandro Bruno, Leo DiCarlo, Nathan Langford, Adrian ParraRodriguez, and Marios Kounalakis for useful discussions. This project has received funding from the Dutch Foundation for Fundamental Research on Matter (FOM), the European Research Council (ERC) under the European Union's Horizon 2020 research and innovation program (Grant Agreement No. 681476-QOMD), and from the Netherlands Organisation for Scientific Research (NWO) in the Innovational Research Incentives Scheme-VIDI, Project No. 680-47-526." The Acknowledgments have been corrected as of 18 December 2018. The Acknowledgments are incorrect in the printed version of the journal. 